\title{
Characterization and validation of an alternative reference bacterium Korean Pharmacopoeia Staphylococcus aureus strain
}

\author{
Ye Won An ${ }^{1}$, Young Sill Choi ${ }^{1}$, Mi-ran Yun ${ }^{2}$, \\ Chihwan $\mathrm{Choi}^{2}$, and Su Yeon Kim ${ }^{1 \star}$ \\ ${ }^{1}$ Division of Pathogen Resource Management, Center for Public Vaccine \\ Development Support, National Institute of Infectious Diseases, National \\ Institute of Health (NIH), Korea Disease Control and Prevention \\ Agency, Cheongju 28160, Republic of Korea \\ ${ }^{2}$ Division of Zoonotic and Vector Borne Disease Research, Center for \\ Infectious Diseases Research, National Institute of Health (NIH), Korea \\ Disease Control and Prevention Agency, Cheongju 28160, Republic of \\ Korea \\ (Received Jun 25, 2021 / Revised Nov 4, 2021 / Accepted Nov 26, 2021)
}

The National Culture Collection of Pathogens (NCCP) is a microbial resource bank in Korea that collects pathogen resources causing infectious disease in human and distributes them for research and education. The NCCP bank attempts to discover strains with various characteristics and specific purposes to provide diverse resources to researchers. Staphylococcus aureus American Type Culture Collection (ATCC) $6538 \mathrm{P}$ is used as a reference strain in the microbial assay for antibiotics in the Korean and in the United States Pharmacopoeias. We aimed to analyze domestically isolated microbial resources from the NCCP to replace the S. aureus reference strain. Staphylococcus aureus strains were identified using matrix-assisted laser desorption/ionization time-of-flight mass spectrometry and the VITEK-2 system and characterized by multilocus sequence typing, $16 \mathrm{~S}$ rRNA sequencing, and antibiotic susceptibility testing. Several candidate strains had similar characteristics as the reference strain. Among them, the nucleotide sequence of the 16S rRNA region of NCCP 16830 was $100 \%$ identical to that of the reference strain; it was sensitive to six types of antibiotics and showed results most similar to the reference strain. A validity evaluation was conducted using the cylinder-plate method. NCCP 16830 presented valid results and had the same performance as ATCC 6538 ; therefore, it was selected as an alternative candidate strain.

Keywords: reference strain, alternative strain, Pharmacopeia, microbial characteristic, Staphylococcus aureus

\section{Introduction}

After adopting the Protocol on Access to Genetic Resources

${ }^{*}$ For correspondence. E-mail: tenksy@korea.kr; Tel.: +82-43-913-4251;

Fax: +82-43-913-4299

Copyright (C) 2022, The Microbiological Society of Korea and Profit Sharing in Nagoya, access to genetic resources has restricted; furthermore, financial or non-monetary profits must be shared when selling products produced from genetic resources (Buck and Hamilton, 2011). The health industry, such as pharmaceuticals and cosmetics, depends heavily on foreign genetic resources. Therefore, some countries including Korea incur additional costs arise in some countries, and they and are continuously discussing measures to reduce these costs. The Korean Pharmacopoeia (KP) contents are derived from the United States Pharmacopeia (USP) and are almost identical. The pharmacopeia contains general test methods for analyzing the safety of preparations and containers for use and includes methods involving microbial resources currently in use. The test reference strain must be used from specific resource banks, including the American Type Culture Collection (ATCC), which is also the case in Korea. For this reason, Korea has to pay for difficult procedures of importing and utilizing foreign strains and economic cost. However, suppose the characteristics of the microbial resources isolated from Korea are investigated and found to be functionally identical to those of the currently used microbial resources currently in use. In that case, these could be used in test methods instead of, for example, the ATCC 6538P reference strain, which is used in the pharmacopeia for the microbial assay for antibiotics. Thus, we characterized numerous strains in this study to identify a Korean strain that could be used as a reference instead of the ATCC strain. Molecular biology analyses and biochemical identification were carried out using matrix-assisted laser desorption/ionization time-of-flight mass spectrometry (MALDI-TOF MS) and VITEK-2. The sensitivity of candidate strains to the major antibiotics was confirmed using the disk diffusion method according to the Clinical and Laboratory Standards Institute (CLSI) guidelines. In addition, genotyping was carried out using multilocus sequence typing (MLST) analysis. After sequencing the $16 \mathrm{~S}$ rRNA, the homology was compared between the reference strain and alternative candidate strains was compared. The homology between the entire genomes was also investigated using whole genome analysis through next-generation sequencing (NGS). After characterizing various candidate strain factors, the strains with the most similar characteristics were selected and used in the actual test method. We confirmed that these strains could be a substitute for the ATCC reference strain. The candidate strains selected through such a survey method could be included in the KP. This would reduce the money and time spend on importing microbial resources. 


\section{Materials and Methods}

\section{Strain isolation}

Staphylococcus aureus ATCC 6538P is a representative reference strain used in the microbial assay for antibiotics a general test method in the KP. This strian was purchased from ATCC (Connolly et al., 1994). Alternative candidate resources included strains clinically isolated strains in Korea and resources collected by the National Culture Collection of Pathogens (NCCP). These strains were isolated and collected from the three local base banks of the NCCP (Jeonbuk National University, Kyungpook National University, and Gyeongsang National University); they originated from blood, urine, feces, the vagina, and cotton swabs scraped from the mucous membranes. Before registering a collected strain in the NCCP, the strain is assigned a management number, combined with a local bank number and specific $\mathrm{P}$-code value, is assigned to the strain, and when it is officially registered in the bank, it is assigned a specific NCCP number. The NCCP has collected $S$. aureus strains. Because of the large number of $S$. aureus candidate strains, 30 strains were randomly selected based on their clinical information, including the region from which they were isolated, the age of patients, source of separation, and separation date. Strain NCCP 16830 was isolated from the urine of a patient in Kyungpook National University (KBN) Hospital in Daegu, Gyeongsangbuk-do, South Korea $\left(35^{\circ} 51^{\prime} 58.7^{\prime \prime} \mathrm{N}, 128^{\circ} 36^{\prime} 16.1^{\prime \prime} \mathrm{E}\right)$. Information on $S$. aureus NCCP 16830 was obtained from literature and the GenBank database (Accession CP043844; http://www.ncbi. nlm.nih.gov/genbank/) and was registered with the NCCP; the NCCP number was received after the completion of the experiment. The strains were grown on $5 \%$ sheep blood agar plates at $37^{\circ} \mathrm{C}$ for $24 \mathrm{~h}$ and preserved at $-80^{\circ} \mathrm{C}$ in $10 \%$ glycerol. Staphylococcus aureus ATCC 6538P is used in the microbial assay for antibiotics in the KP and USP. The test was conducted using the cylinder-plate method by growing $S$. aureus in a plate containing neomycin sulfate.

\section{Identification}

For bacterial identification, the morphology of the colony, including color, and hemolysis type, was confirmed. MALDITOF MS analysis was performed using Biotyper software (BrukerDaltonik) to identify bacteria (Singhal et al., 2015).

\section{Biochemical and molecular characterization}

(i) To analyze the biochemical reactions and experiments were performed according to the manufacturer's manual using a VITEK 2 GP card (bioMérieux) (Delmas et al., 2008). The susceptibility or resistance of the strains to antibiotics was assessed using the disk diffusion method, according to the CLSI guidelines (CLSI, 2019).

(ii) Homology of the $16 \mathrm{~S}$ rRNA sequence was confirmed by global alignment (National Center for Biotechnology Information, NCBI) of the $16 \mathrm{~S}$ rRNA sequence of each reference strain and candidate strain (Lane, 1991; Chun et al., 2007). The results are shown as percent homology.

(iii) MLST was performed according to the method described by Enright et al. (2000). The allele sequences of seven genes were compared using the Pubmlst.org database, and the number of alleles was determined. The sequence number (ST) was determined by combining the allele numbers of the seven genes (Enright et al., 2000).

\section{Whole genome analysis using NGS}

NGS was performed using PacBio RSII (Pacific Biosciences) and Ion S5 (Thermo Fisher Scientific) to analyze the homology of the full-length sequence was analyzed using the OrthoANI program (Lee et al., 2016). Multiple sequence alignment was performed using Mauve aligner for genome structure analysis (Darling et al., 2004). Sequences were subjected to de novo assembly using the Hierarchical Genome Assembly Process 3 and FALCON, and the final whole genome sequence was determined by correcting sequencing errors using Proovread (v2.14) for parts of the contig with a low sequence quality. The complete nucleotide sequence and genome metadata of each strain have been registered with the NCBI.

\section{Validity testing}

The General test which is one of the components of the KP, describes common test methods, reagents, and test solutions, standard solutions and containers for the drugs listed in the formulary. The cylinder-plate method, Standard curve method, and Turbidimetric methods are used for the assay of individual monograph. The five plates that have been tested are considered as a group (Fig. 1). One plate was divided into four fractions, and the cylinder place at the center of one fraction. In the four cylinders, low and high concentration standard solutions were and low and high concentration sample solutions are injected and incubated. After culturing, measure the diameter of the growth-inhibiting zone where microorganisms do not grow by antibiotics based on the cylinder was measured. The results were calculated and derived according to the titer calculation method presented in KP's test method.

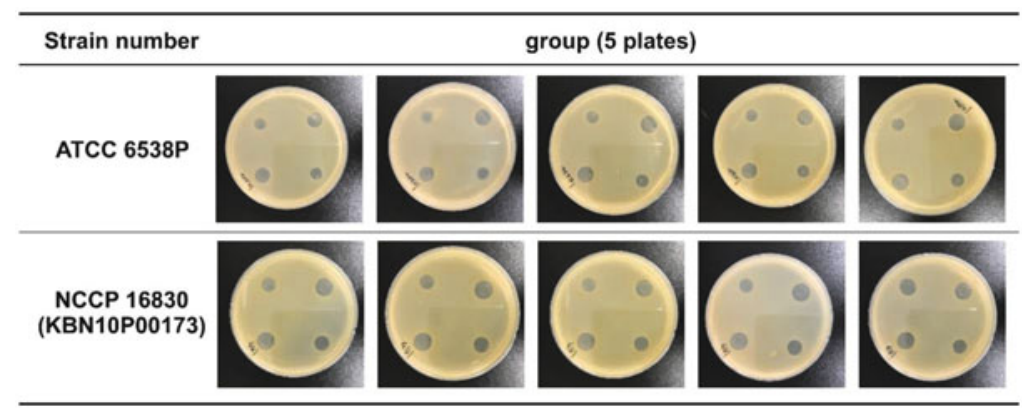

Fig. 1. The validity test for Staphylococcus aureus using the cylinder-plate method. This figure is the result of usability evaluation by applying the cylinder-plate method. The four circles you see inside the plate are where the cylinder used to be. When an antibiotic is injected into the cylinder and then cultured, the growth of microorganisms is suppressed and a clear zone is formed. Calculate the antibiotic titer by measuring the size of this clear zone. 
Validity test confirmed that the performance is similar between the two strains by experimenting using a reference strain and a strain isolated from Korea as a bacterial suspension in these test methods. Validity test was also conducted by the cylinder-plate method. All experiments were performed according to the general test and quantification methods of $\mathrm{KP}$. The average titer was calculated from five plates in one group, and the same experiment was repeated 10 times to obtain the average value of all experimental values. The effective range was established by calculating \pm 2 standard deviations (SD) from the mean titer of the reference strain. The average titer of the candidate strain was calculated to confirm that it was within the effective range. The effective range criteria for deriving the results were established according to the method stipulated in the $\mathrm{R} \& \mathrm{D}$ report of the Korean Ministry of Food and Drug Safety in Korea in 2018.

\section{Results}

\section{Phenotype and characteristics}

(i) All S. aureus strains formed yellow colonies with diameter of less than $0.5 \mathrm{~mm}$ in diameter and showed $\beta$-hemolytic activity. Thirty NCCP S. aureus candidate strains and ATCC
6538P were identified as $S$. aureus using MALDI-TOF MS with a score of 2.0 or higher (Table 1). The VITEK-2 analysis was performed to characterize and identify the strains, the 30 candidate strains, two were identified as $S$. aureus with $95 \%$ accuracy, one with $97 \%$, two with $98 \%$, and 25 with $99 \%$ accuracy (Table 1).

(ii) MLST was performed for all the strains, including the reference strain. Results were only obtained for only 21 candidate strains and the reference strain. ATCC 6538P had an ST of 464, and none of the candidate strains had the same ST number (Table 1).

(iii) Global alignment was performed using the 16S rRNA sequence of the reference strain and each candidate strain; the genetic homology between the strains was compared and expressed as a percentage (\%). Sixteen strains had 100\% homology to the reference strain (Table 1). The reference strain and the 30 randomly selected candidate strains were identified and characterized, and the experimental results were compared. Among the strain numbers in Table 1, NCCP number indicates a registered resource with the bank, and KBN number indicates a resource that has been collected and held but not yet registered.

(iv) The susceptibility and resistance to six antibiotics of the 16 strains showing $100 \% 16 \mathrm{~S}$ rRNA homology were confirmed using the disk diffusion method. The reference strain

Table 1. Results of Staphylococcus aureus identification and characterization

\begin{tabular}{|c|c|c|c|c|c|}
\hline Sample number & Morphological shape & MLST (ST no.) & MALDI-TOF (score value) & VITEK-2 (\%) & 16S rRNA similarity (\%) \\
\hline ATCC 6538P & \multirow{31}{*}{$\begin{array}{l}\text { Gram-positive cocci, } \\
0.5 \mathrm{~mm} \text { in diameter, yellow, } \\
\text { sporulation negative, } \\
\beta \text {-hemolysis positive }\end{array}$} & 464 & 2.512 & 99 & - \\
\hline KBN10P00169 & & - & 2.407 & 99 & 100.00 \\
\hline KBN10P00172 & & 72 & 2.392 & 99 & 100.00 \\
\hline NCCP16830 & & 72 & 2.442 & 99 & 100.00 \\
\hline KBN12P00057 & & - & 2.381 & 99 & 99.87 \\
\hline KBN12P00073 & & 72 & 2.311 & 99 & - \\
\hline KBN12P00101 & & - & 2.311 & 99 & 100.00 \\
\hline KBN10P00529 & & 72 & 2.266 & 99 & 99.87 \\
\hline KBN10P00548 & & 72 & 2.456 & 98 & 99.93 \\
\hline KBN12P00577 & & 188 & 2.488 & 99 & 100.00 \\
\hline KBN12P00582 & & 1 & 2.442 & 99 & 99.93 \\
\hline KBN12P00600 & & - & 2.465 & 99 & 100.00 \\
\hline KBN12P00602 & & - & 2.346 & 99 & 99.87 \\
\hline KBN12P00609 & & 15 & 2.297 & 99 & 99.93 \\
\hline KBN12P00622 & & 513 & 2.558 & 98 & 99.93 \\
\hline KBN12P01374 & & 188 & 2.562 & 99 & 99.93 \\
\hline KBN12P01369 & & 72 & 2.441 & 99 & 100.00 \\
\hline KBN12P01343 & & - & 2.591 & 99 & 99.93 \\
\hline KBN12P02380 & & 72 & 2.331 & 99 & 100.00 \\
\hline KBN12P02382 & & 72 & 2.562 & 99 & 99.93 \\
\hline KBN12P02401 & & 97 & 2.358 & 95 & 100.00 \\
\hline KBN12P02402 & & 72 & 2.470 & 97 & 99.93 \\
\hline KBN12P02409 & & - & 2.329 & 99 & 100.00 \\
\hline KBN06P01503 & & 72 & 2.426 & 99 & - \\
\hline KBN06P01542 & & 72 & 2.431 & 95 & 100.00 \\
\hline KBN06P01593 & & 72 & 2.499 & 99 & 100.00 \\
\hline KBN06P01660 & & - & 2.417 & 99 & 100.00 \\
\hline KBN06P01716 & & 5 & 2.467 & 99 & 100.00 \\
\hline KBN06P01783 & & 72 & 2.442 & 99 & 100.00 \\
\hline KBN06P04841 & & - & 2.386 & 99 & 100.00 \\
\hline KBN06P04845 & & 5 & 2.434 & 99 & 99.93 \\
\hline
\end{tabular}

ST no., sequence type number. 
Table 2. Antibiotic susceptibility testing using the disk diffusion method

\begin{tabular}{lcccccc}
\hline \multirow{2}{*}{ Strain number } & \multicolumn{6}{c}{ Antibiotic } \\
\cline { 2 - 7 } & $\begin{array}{c}\text { Azithro- } \\
\text { mycin }\end{array}$ & $\begin{array}{c}\text { Erythro- } \\
\text { mycin }\end{array}$ & $\begin{array}{c}\text { Clinda- } \\
\text { mycin }\end{array}$ & Oxacillin & Cefoxitin & $\begin{array}{c}\text { Genta- } \\
\text { micin }\end{array}$ \\
\hline ATCC 6538P & S & S & S & S & S & S \\
NCCP 16830 & S & S & S & S & S & S \\
KBN12P00600 & S & S & S & S & S & S \\
KBN06P01542 & R & R & R & R & R & S \\
KBN06P01593 & R & R & R & R & R & R \\
KBN12P02401 & S & S & S & S & R & R \\
KBN12P02409 & S & S & S & S & R & S \\
KBN10P00169 & S & S & S & S & R & S \\
KBN10P00172 & S & S & S & I & R & S \\
KBN12P00101 & S & S & S & S & R & S \\
KBN06P01660 & S & S & S & S & R & R \\
KBN06P01716 & R & R & R & R & S & R \\
KBN06P01783 & R & R & R & R & S & S \\
KBN06P04841 & R & S & S & R & R & S \\
KBN12P00577 & S & S & S & S & R & S \\
KBN12P01369 & S & S & S & S & R & S \\
KBN12P02380 & S & S & S & I & R & S \\
\hline
\end{tabular}

$\mathrm{R}$, resistant; $\mathrm{S}$, sensitive.

ATCC 6538P showed sensitivity to all the six antibiotics tested (azithromycin, erythromycin, clindamycin, oxacillin, cefoxitin, and gentamicin). The candidate strains NCCP 16830 and KBN12P00600 showed the same results. The final candidate strain was selected based on its usefulness (Table 2).

\section{Validations}

The average titer was obtained 10 times using the microbial assays for antibiotics with ATCC 6538P and by measuring the size of the obtained clear zone. The calculated average titer was $116.62 \%$, and with an SD of this titer was $12.28 \%$. Therefore, the set effective range was $92.059 \%<$ average titer of candidate strain $<141.178 \%$. The average titer obtained from the 10 replicates of the candidate strain NCCP 16830 was 120.34, and the average titer of KBN12P00600 was 355.41. Thus, the average titer of NCCP 16830 was within the effective range, but the average titer of KBN12P00600 was not within the effective range (Table 3 ).

\section{Comparative genomics}

Complete genomic sequences of ATCC 6538P and NCCP 16830 have been registered with GenBank under accession numbers CP041746 and CP043843, respectively. The fulllength sequence homology between the reference strain and the alternative candidate strain was analyzed using the OrthoANI program. The sequence similarity of sequences between
Table 3. Validity evaluation results and effective range

\begin{tabular}{ccc}
\hline \multirow{2}{*}{ Experiment number } & \multicolumn{2}{c}{ Neomycin sulfate titer (\%) } \\
\cline { 2 - 3 } 1 & ATCC 6538P & NCCP 16830 \\
\hline 2 & 130.5822 & 123.8903 \\
3 & 119.3446 & 127.4353 \\
4 & 94.68126 & 110.2635 \\
5 & 121.7437 & 116.2017 \\
6 & 103.8965 & 113.6195 \\
7 & 129.1561 & 117.5756 \\
8 & 127.4382 & 113.9225 \\
9 & 114.8647 & 120.9919 \\
10 & 103.0279 & 124.3137 \\
Average titer & 121.4486 & 135.1476 \\
Standard deviation (SD) & 116.6184 & 120.3362 \\
SD * 2 & 12.27981 & 7.535702 \\
Average titer - 2 SD & 24.55963 & - \\
Average titer + 2 SD & 92.059 & - \\
\hline
\end{tabular}

ATCC 6538P and NCCP 16830 sequences was $99.00 \%$. The reference and candidate strains consisted of five structural blocks, and the nucleotide sequence within the block was well preserved, as shown in Fig. 2. There was an additional genomic region in the $1,980-2,020 \mathrm{~kb}$ of strain NCCP16830. This insertion is not found in the reference genome. The major genomic difference was composed of 34 CDS with 18 hypothetical genes and one pseudogene.

\section{Discussion}

The safety of ingredients or containers during the development and production of food and pharmaceutical products is evaluated using country-specific certification tests. The test products must pass established standards, including experiments using pathogenic microorganisms. The pathogenic microorganisms used in these tests should be obtained from a specific microbial bank; the KP also states that foreign resource banks, such as the ATCC, should be used to procure these microorganisms. The contents of the KP were compiled from the USP. Although the test methods stipulated in the pharmacopeias slightly differ by country, the methods are partially annually for international harmony and export expansion.

Several companies in Korea are conducting experiments using test methods and materials that meet these standards. However, as there are no isolated microbial resources in the country, reference strains must be imported. Furthermore, the implementation of the Nagoya Protocol has complicated the procedure for importing foreign strains has become more

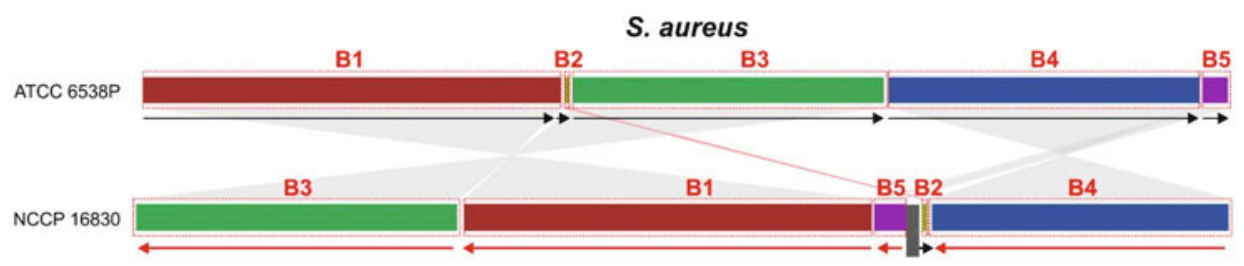

Fig. 2. Multi sequence alignment using Mauve aligner. Multi genome alignment of NCCP16830 genome with the reference ATCC strain genome using Mauve aligner. Linked colored blocks indicate the homologous segments in each genome. Arrows show orientation of each segment. 
complicated, thereby increasing the time and cost required, which burdens domestic researchers.

The USP states that it can be used replacement strains that has been proven to be equivalent to a reference strain (United States Pharmacopeia, 2020). Therefore, this study was conducted to identify a strain from Korea with the same performance as the existing reference strain, for utilization use as a reference.

The most representative strain of $S$. aureus, which is used in the microbial assay of antibiotics in the general test methods of the KP and USP, is ATCC 6538P (Oh et al., 2009; MFDS, 2014). Characteristic comparative experiments were conducted to replace the ATCC strain with NCCP strains isolated in Korea. The results of the comparison between the reference and candidate strains revealed that the morphological, biochemical, and molecular characteristics of the strains were the same. Moreover, whole-genome sequence comparison revealed a high homology of $99 \%$. While the sequence identity of whole genomes between two strains is $99 \%$, the inserted region in the NCCP16830 genome was disclosed as the major genomic difference was disclosed by MauveAligner and COG analysis. In this region, the functions of 18 out of 34 genes were predicted their functions. Six genes were belonged to six categories D, E, F, G, K, and M, respectively. Each two of the eight genes were predicted to four categories, P, R, S, and $\mathrm{X}$. The last four genes were belonged to categories $\mathrm{L}$. Of these genes, five were distributed to the category Information Storage and Processing, two to the category Cellular Processes and Signaling, and 11 to category Metabolism. Further detailed functions and impacts of these genes have to be studied experimentally. However, even with the same characteristics, the strain would not be qualified as an alternative strain if the test results differed from those of the reference strain.

This test method measured the antibiotic susceptibility titer of drugs using microorganisms to meet the acceptance criteria. Therefore, the microorganisms used should not be resistant to antibiotics. Similar to the ATCC strain, NCCP 16830 was sensitive to major antibiotics, including neomycin sulfate and the other antibiotics, used in the test; and it showed almost the same susceptibility titer as the ATCC strain.

Suppose a strain that presents the same performance as the reference strain, such as NCCP 16830, is registered as an equivalent reference genome strain and researchers use it as an alternative. In that case, the cost and time required to import resources will be substantially reduced, and the degree of foreign dependence will decrease.

\section{Acknowledgements}

This study was funded by the Korea Disease Control \& Prevention Agency, Ministry of Health and Welfare, grant numbers 2017-NG45005-00 and 2019-NG-047-00.

\section{Conflict of Interest}

The authors declare no conflicts of interest.

\section{Author Contributions}

Conceptualization, S.Y.K.; methodology, Y.W.A.; software, Y.W.A.; validation, S.Y.K., Y.W.A., Y.S.C., M.Y., and C.C.; formal analysis, S.Y.K.; investigation, S.Y.K., Y.W.A., and Y.S.C.; resources, S.Y.K.; data curation, Y.W.A.; writing-original draft preparation, Y.W.A.; writing-review and editing, S.Y.K.; visualization, Y.W.A.; supervision, S.Y.K. and Y.S.C.; project administration, S.Y.K. and Y.W.A.; funding acquisition, S.Y.K. and Y.S.C. All authors have read and agreed to the published version of the manuscript.

\section{Data Availability Statement}

The data used in this study are available in this article.

\section{References}

Buck, M. and Hamilton, C. 2011. The Nagoya Protocol on access to genetic resources and the fair and equitable sharing of benefits arising from their utilization to the convention on biological diversity. Rev. Eur. Comp. Int. Environ. Law 20, 47-61.

Chun, J., Lee, J.H., Jung, Y., Kim, M., Kim, S., Kim, B.K., and Lim, Y.W. 2007. EzTaxon: a web-based tool for the identification of prokaryotes based on $16 \mathrm{~S}$ ribosomal RNA gene sequences. Int. J. Syst. Evol. Microbiol. 57, 2259-2261.

CLSI, Clinical and Laboratory Standards Institute. 2019. Performance Standards for Antimicrobial Susceptibility Testing. 29th edn, pp. 34-44. CLSI supplement M100. Wayne, Pennsylvania, USA.

Connolly, P., Bloomfield, S.F., and Denyer, S.P. 1994. The use of impedance for preservative efficacy testing of pharmaceuticals and cosmetic products. J. Appl. Bacteriol. 76, 68-74.

Darling, A.C.E., Mau, B., Blattner, F.R., and Perna, N.T. 2004. Mauve: multiple alignment of conserved genomic sequence with rearrangements. Genome Res. 14, 1394-1403.

Delmas, J., Chacornac, J.P., Robin, F., Giammarinaro, P., Talon, R., and Bonnet, R. 2008. Evaluation of the Vitek 2 system with a variety of Staphylococcus species. J. Clin. Microbiol. 46, 311-313.

Enright, M.C., Day, N.P., Davies, C.E., Peacock, S.J., and Spratt, B.G. 2000. Multilocus sequence typing for characterization of methicillin-resistant and methicillin-susceptible clones of Staphylococcus aureus. J. Clin. Microbiol. 38, 1008-1015.

Lane, D.J. 1991. 16S/23S rRNA sequencing. In Stackebrandt, E. and Goodfellow, M. (eds.), Nucleic Acid Techniques in Bacterial Systematic, pp. 115-175. John Wiley \& Sons, New York, USA.

Lee, I., Kim, Y.O., Park, S.C., and Chun, J. 2016. OrthoANI: An improved algorithm and software for calculating average nucleotide identity. Int. J. Syst. Evol. Microbiol. 66, 1100-1103.

MFDS, Ministry of Food and Drug Safety. 2014. The Korean Pharmacopoeia, 11th edn., pp. 2095-2392. Ministry of Food and Drug Safety, Republic of Korea.

Oh, H.Y., Kim, D.S., Kim, Y.O., Yoo, M.Y., Lee, S., Sohn, K.H., Um, S.Y., Kim, D.H., Kim, T.S., and Oh, I.U. 2009. Introduction of worldwide organizations and systems of Pharmacopoeia. Regul. Res. Food Drug Cosmet. 4, 29-38.

Singhal, N., Kumar, M., Kanaujia, P.K., and Virdi, J.S. 2015. MALDITOF mass spectrometry: an emerging technology for microbial identification and diagnosis. Front. Microbiol. 6, 791.

United States Pharmacopeia. 2020. Antibiotics-Microbial Assays, USP43-NF38 - 6488, pp. 44. The Unites States Pharmacopoeia, Maryland, USA. 